\title{
Complex Dynamics of a Ratio-Dependent Predator-Prey Model Induced by Spatial Motion
}

\author{
Caiyun Wang $\mathbb{D}^{1}{ }^{1}$ Jing $\mathrm{Li}^{2}$ and Ruiqiang $\mathrm{He}^{1}$ \\ ${ }^{1}$ Department of Mathematics, Xinzhou Teachers University, Xinzhou, Shanxi 034000, China \\ ${ }^{2}$ Shanxi Universtity of Finance and Economics, Taiyuan, Shanxi 034006, China \\ Correspondence should be addressed to Caiyun Wang; 494423138@qq.com
}

Received 25 November 2020; Revised 13 February 2021; Accepted 20 February 2021; Published 11 March 2021

Academic Editor: Quanmin Zhu

Copyright ( 12021 Caiyun Wang et al. This is an open access article distributed under the Creative Commons Attribution License, which permits unrestricted use, distribution, and reproduction in any medium, provided the original work is properly cited.

One of the most efficient predator-prey models with spatial effects is the one with ratio-dependent functional response. However, there is a need to further explore the effects of spatial motion on the dynamic behavior of population. In this work, we study a ratio-dependent predator-prey model with diffusion terms. The aim of this work is to investigate the changes in predator's distribution in space as the prey populations change their mobility. We observe that the frequency diffusion of the prey gives rise to the sparse density of the predator. Moreover, we also observe that the increasing rate of the conversion into predator biomass induces pattern transitions of the predator. Specifically speaking, Turing pattern of the predator populations goes gradually from a spotted pattern to a black-eye pattern, with the intermediate state being the mixture of spot and stripe pattern. The simulation results and analysis of this work illustrate that the diffusion rate and the real intrinsic factor influence the persistence of the predator-prey system mutually.

\section{Introduction}

The dynamical behavior of the prey-predator model has been investigated by researchers for many years after the pioneering work was accomplished by Lotka and Volterra. The authors present the well-known Lotka-Volterra predator-prey model. It is established that the response function is the key element in the predator-prey models. The ratio-dependent functional response was first proposed by Leslie and Gower in 1973 [1,2]. The authors discuss that the predator's growth is described by prey's density, as well as by the ratio of density of the predator to prey. Afterwards, the Holling-Tanner rate in the predator-prey model is presented in the literature. This model is also known as the Holling-Tanner predator-prey model [3]. The dynamics such as local and global stability, limit cycles, and pattern solutions of Holling-Tanner predator-prey model have already been investigated [4-7]. The factors such as delay and random are also considered in the recent literature [8-13]. The fuzzy approach is also considered to construct the predator-prey model $[14,15]$. However, the
Holling-Tanner-type predator-prey models are important because of their appropriateness for modeling the real ecological interactions between predator and prey species. The Holling-Tanner predator-prey model is

$$
\left\{\begin{array}{l}
\frac{\mathrm{d} N}{\mathrm{~d} T}=\mathrm{RN}\left(1-\frac{N}{K}\right)-\frac{\mathrm{MNP}}{N+D}, \\
\frac{\mathrm{d} P}{\mathrm{~d} T}=P \Theta\left(1-\frac{\mathrm{HP}}{N}\right),
\end{array}\right.
$$

where $N(T)$ and $P(T)$ denotes the prey and predator density, respectively, at time T.R, $K, \Theta, M, D$, and $H$ are all positive, and their definitions are given in Table 1.

The term MNP/N + D in model (1) is known as Holling type II response function [16] which was proposed by Holling in 1965. In addition, it is assumed that the functional response is expressed by Holling type III response function [17-19], which is more suitable for the population of vertebrates. The ratio-dependent predator-prey model with Holling type III functional response is 
TABLE 1: The definition of parameters used in model (1).

\begin{tabular}{lc}
\hline Parameter & Meaning \\
\hline$K$ & The prey's intrinsic growth rate \\
\hline$M$ & The carrying capacity of the prey \\
\hline$H$ & The largest amount of prey that predator can capture per unit time \\
\hline$D$ & Predator intrinsic growth rate \\
\hline
\end{tabular}

$$
\left\{\begin{array}{l}
\frac{\mathrm{d} N}{\mathrm{~d} T}=\mathrm{RN}\left(1-\frac{N}{K}\right)-\frac{M N^{2} P}{A N^{2}+1}, \\
\frac{\mathrm{d} P}{\mathrm{~d} T}=P \Theta\left(1-\frac{\mathrm{HP}}{N}\right),
\end{array}\right.
$$

where $A$ is the half-saturation constant.

On the other hand, there are a large number of organisms with spatial pattern distributions [20-22]. The distribution of steady spatial pattern is called Turing pattern. This model was put forward when Turing presented the theory of mathematical explanation for the pattern formation of the animal skin [23]. Following Turing's famous work, the researchers have already explained self-organized pattern formation in various fields such as geology, physics, chemistry, and biology [20-28]. Especially, for the diffusive predator-prey model in ecology, pattern formation helps us in a better way to understand the influence of predator's individual mobilities, the dynamics of stability, and oscillations of the prey species. There are various works that present the spatial predator-prey models [7, 29-35]. Wu and Song discussed the joint impact of nonlocal term and delay on the spatial predator-prey model [36]. Cao and Jiang indicate that the nonlocal reaction term can stabilize the spatially inhomogeneous periodic solutions [37]. Incorporating the diffusive terms into model (2), we have

$$
\left\{\begin{array}{l}
\frac{\partial N}{\partial T}=\mathrm{RN}\left(1-\frac{N}{K}\right)-\frac{M N^{2} P}{A N^{2}+1}+D_{1} \nabla^{2} N, \quad(x, T) \in \Omega \times(0, \infty) \\
\frac{\partial P}{\partial T}=\Theta P\left(1-\frac{H P}{N}\right)+D_{2} \nabla^{2} N, \quad(x, T) \in \Omega \times(0, \infty) \\
\frac{\partial N(x, T)}{\partial n}=\frac{\partial P(x, T)}{\partial n}=0, \quad(x, T) \in \partial \Omega \times(0, \infty) \\
N(x, 0)=N_{0}>0, \quad x \in \Omega \\
P(x, 0)=P_{0}>0, \quad x \in \Omega
\end{array}\right.
$$

where $D_{11}$ and $D_{22}$ represent the diffusion coefficients of the prey and predator, respectively, which are all positive.

The mathematical analysis of the spatial predator-prey model used to be based on linear stability analysis. However, linear stability analysis is only meaningful in the small domain of the equilibrium, where the linear system is approximated to nonlinear systems. The multiple scale method [38-40] is employed to study spatial dynamic behaviors of the model in our present paper. This method is effective when the system under consideration is a partial differential equation. Specifically, the parameters in system are decomposed into different scales. The amplitude equation is an important tool to understand the pattern selection near the Turing bifurcation boundary. By solving the amplitude equation, we obtain the domain of the controlled parameter, where the steady state pattern arises. The rest of our paper is organized as follows.
In Section 2, we present an example to show the conditions for the occurrence of Turing instability by bifurcation analysis. In Section 3, we derive the amplitude equations of our model on the basis of multiple scale analysis. In Section 4 , we present the simulations performed to illustrate the different patterns in the corresponding Turing domain. Moreover, the predator's pattern transitions driven by prey's diffusion rate and conversation rate of biomass into predator are discussed in detail. Finally, in Section 5, we conclude our work.

\section{Bifurcation Analysis}

In this section, we present the conditions for the occurrence of Turing pattern. We choose the scale $\widetilde{u}=N / K, \widetilde{v}=\mathrm{MKP} /$ $R$, and $\widetilde{t}=\mathrm{RT}$, and we obtain 


$$
\left\{\begin{array}{l}
\frac{\partial \widetilde{u}}{\partial \widetilde{t}}=\widetilde{u}(1-\widetilde{u})-\frac{\widetilde{u}^{2} \widetilde{v}}{e \widetilde{u}^{2}+\widetilde{v}}+d_{1} \nabla^{2} \widetilde{u}, \quad(x, \widetilde{t}) \in \Omega \times(0, \infty), \\
\frac{\partial \widetilde{v}}{\partial \widetilde{t}}=\widetilde{v} \eta\left(1-\frac{\gamma \widetilde{v}}{\widetilde{u}}\right)+d_{2} \nabla^{2} \widetilde{v}, \quad(x, \widetilde{t}) \in \Omega \times(0, \infty), \\
\frac{\partial \widetilde{u}(x, \widetilde{t})}{\partial n}=\frac{\partial \widetilde{v}(x, \widetilde{t})}{\partial n}=0, \quad(x, t) \in \partial \Omega \times(0, \infty), \\
\widetilde{u}(x, 0)=u_{0}>0, \quad x \in \Omega, \\
\widetilde{v}(x, 0)=v_{0}>0, \quad x \in \Omega,
\end{array}\right.
$$

where $\eta=\Theta / R, e=A K^{2}, \gamma=\mathrm{HR} / \mathrm{MK}^{2}, d_{1}=\mathrm{KD}_{1} / R$, and $d_{2}$ $=\mathrm{RD}_{2} / M^{2} K^{3}$.

The analysis of the existence and local stability of the positive equilibrium is presented in [41]. Here, we present an example to illustrate the existence and local stability of the positive equilibrium. Let $E^{*}\left(\widetilde{u}^{*}, \widetilde{v}^{*}\right)$ be an interior steady equilibrium of model (4). The Jacobin matrix of model (4) is

$$
\begin{gathered}
J=\left(\begin{array}{cc}
f_{\widetilde{u}} & f_{\widetilde{v}} \\
g_{\widetilde{u}} & g_{\widetilde{v}}
\end{array}\right), \\
\widetilde{u}(1-\widetilde{u})-\frac{\widetilde{u}^{2} \widetilde{v}}{e \widetilde{u}^{2}+1} \triangleq f(\widetilde{u}, \widetilde{v}), \widetilde{v} \eta\left(1-\frac{\gamma \widetilde{v}}{\widetilde{u}}\right) \triangleq g(\widetilde{u}, \widetilde{v}),
\end{gathered}
$$

and the trace and determinant of matrix $J$ are

$$
\begin{aligned}
\operatorname{tr}(J) & =\frac{-1+e\left(\widetilde{u}^{*}\right)^{2}-2 e\left(\widetilde{u}^{*}\right)^{3}}{1+e\left(\widetilde{u}^{*}\right)^{2}}-\eta, \\
\operatorname{det}(J) & =\frac{\eta \widetilde{u}^{*}}{1+e\left(\widetilde{u}^{*}\right)^{2}} F^{\prime}\left(\widetilde{u}^{*}\right) .
\end{aligned}
$$

On the basis of parameters presented in Table 2, we calculate the equilibrium and get the stability of the $E^{*}\left(\widetilde{u}^{*}, \widetilde{v}^{*}\right)$.

The characteristic polynomial at $E^{*}\left(\widetilde{u}^{*}, \widetilde{v}^{*}\right)$ is

$$
\left|\lambda E-J_{k}\right|=0 \text {, }
$$

where

$$
J_{k}=\left(\begin{array}{cc}
f_{\widetilde{u}}-d_{1} k^{2} & f_{\widetilde{v}} \\
g_{\widetilde{u}} & g_{\widetilde{v}}-d_{2} k^{2}
\end{array}\right)
$$

and $k$ is the wave number. The eigenvalue is

$$
\lambda_{k}^{2}-\operatorname{tr}_{k} \lambda_{k}+\Delta_{k}=0,
$$

where

$$
\begin{aligned}
t r_{k} & =\left(f_{\widetilde{u}}+g_{\widetilde{v}}\right)-k^{2}\left(d_{1}+d_{2}\right), \\
\Delta_{k} & =f_{\widetilde{u} g_{\tilde{v}}}-f_{\widetilde{v}} g_{\widetilde{u}}-k^{2}\left(d_{2} f_{\widetilde{u}}+d_{1} g_{v}\right)+d_{1} d_{2} k^{4} \\
& =\operatorname{det}(J)-k^{2}\left(d_{2} f_{\widetilde{u}}+d_{1} g_{\widetilde{v}}\right)+d_{1} d_{2} k^{4} .
\end{aligned}
$$

Therefore, the eigenvalue of (7) is

$$
\lambda_{k}=\frac{t r_{k} \pm \sqrt{t r_{k}^{2}-4 \Delta_{k}}}{2} .
$$

The Hopf bifurcation occurs when the conditions $\operatorname{Re}\left(\lambda_{k}\right)=0$ and $\operatorname{Im}\left(\lambda_{k}\right) \neq 0$ hold at $k=0$. However, Turing bifurcation occurs when conditions $\operatorname{Re}\left(\lambda_{k}\right)=0$ and Im $\left(\lambda_{k}\right)=0$ hold at $k=k_{T}>0$. And, $k_{T}$ satisfies

$$
k_{T}^{2}=\sqrt{\frac{f_{\widetilde{u}} g_{\tilde{v}}-f_{\widetilde{v}} g_{\widetilde{u}}}{d_{1} d_{2}}}=\sqrt{\frac{\operatorname{det} J}{d_{1} d_{2}}} .
$$

Substituting (12) into $\Delta_{k}=0$, we get the expression of $d_{1}^{T}$. The value of this expression represents the critical value of Turing bifurcation parameter. Turing domain and the real part of eigenvalues of model 4 are shown in (Figures 1 and 2).

$$
d_{1}^{T}=\frac{d_{2} f_{\widetilde{u}}}{2\left(f_{\widetilde{u}} g_{\widetilde{v}}-f_{\widetilde{v}} g_{\widetilde{u}}+\sqrt{f_{\widetilde{v}}^{2} g_{\widetilde{u}}^{2}-f_{\tilde{u}} f_{\widetilde{v}} g_{\tilde{u}} g_{\tilde{v}}}\right) / f_{\widetilde{u}}-g_{\mathcal{v}}} .
$$

\section{Multiple Scale Analysis}

We choose $d_{1}$ and $d_{1}^{T}$ as a control parameter and the bifurcation threshold, respectively. This is done in order to deduce the amplitude equations via multiple scale analysis $[42,43]$ and judge the type of the Turing pattern.

Near $d_{1}=d_{1}^{T}$, system (4) is written at $E^{*}\left(\widetilde{u}^{*}, \widetilde{v}^{*}\right)$ as follows:

$$
\frac{\partial U}{\partial t}=\mathrm{LU}+N,
$$

where

$$
U=\left(\begin{array}{l}
\tilde{u} \\
\widetilde{v}
\end{array}\right), L=\left(\begin{array}{cc}
f_{\widetilde{u}}+d_{1} \nabla^{2} & f_{\widetilde{v}} \\
g_{\widetilde{u}} & g_{\widetilde{v}}+d_{2} \nabla^{2}
\end{array}\right),
$$




$$
\tilde{h}_{3}=\left(\begin{array}{c}
\frac{1}{6} p_{111} \tilde{u}_{1}^{3}+\frac{1}{6} p_{222} \widetilde{v}_{1}^{3}+\frac{1}{6}\left(p_{112}+p_{121}+p_{211}\right) \tilde{u}_{1}^{2} \widetilde{v}_{1}+\frac{1}{6}\left(p_{122}+p_{212}+p_{221}\right) \tilde{u}_{1} \widetilde{v}_{1}^{2} \\
+p_{11} \widetilde{u}_{2} \widetilde{u}_{1}+p_{22} \widetilde{v}_{2} \widetilde{v}_{1}+\frac{1}{2}\left(p_{12}+p_{21}\right)\left(\widetilde{u}_{2} \widetilde{v}_{1}+\widetilde{u}_{1} \widetilde{v}_{2}\right) \\
\frac{1}{6} q_{111} \tilde{u}_{1}^{3}+\frac{1}{6} q_{222} \widetilde{v}_{1}^{3}+\frac{1}{6}\left(q_{112}+q_{121}+q_{211}\right) \widetilde{u}_{1}^{2} \widetilde{v}_{1}+\frac{1}{6}\left(q_{122}+q_{212}+q_{221}\right) \tilde{u}_{1} \widetilde{v}_{1}^{2} \\
+q_{11} \widetilde{u}_{2} \widetilde{u}_{1}+q_{22} \widetilde{v}_{2} \widetilde{v}_{1}+\frac{1}{2}\left(q_{12}+q_{21}\right)\left(\tilde{u}_{2} \widetilde{v}_{1}+\widetilde{u}_{1} \widetilde{v}_{2}\right)
\end{array}\right),
$$

where $p_{i j k}$ and $q_{i j k}$ denote the derivative of $i$ th, $j$ th, and $k$ th where variable successively to $f$ and $g$ at $E^{*}\left(\tilde{u}^{*}, \widetilde{v}^{*}\right) \cdot U, t$, and $N$ are expanded with a small parameter $\varepsilon$.

$$
\begin{aligned}
d_{1}^{T}-d_{1} & =\varepsilon d_{11}+\varepsilon^{2} d_{22}+\ldots, \\
\widetilde{t} & =T_{0}+\varepsilon T_{1}+\varepsilon^{2} T_{2}, \\
U & =\left(\begin{array}{c}
\widetilde{u} \\
\widetilde{v}
\end{array}\right) \\
N & =\varepsilon^{2} \widetilde{h}_{2}+\varepsilon^{3} \widetilde{h}_{3}+o\left(\varepsilon^{4}\right),
\end{aligned}
$$

$$
\begin{aligned}
& \tilde{h}_{2}=\left(\begin{array}{c}
\frac{1}{2} p_{11} \widetilde{u}_{1}^{2}+\frac{1}{2}\left(p_{12}+p_{21}\right) \widetilde{u}_{1} \widetilde{v}_{1}+\frac{1}{2} p_{22} \widetilde{v}_{1}^{2} \\
\frac{1}{2} q_{11} \widetilde{u}_{1}^{2}+\frac{1}{2}\left(q_{12}+q_{21}\right) \widetilde{u}_{1} \widetilde{v}_{1}+\frac{1}{2} q_{22} \widetilde{v}_{1}^{2}
\end{array}\right), \\
& \tilde{h}_{3}=\left(\begin{array}{c}
\frac{1}{6} p_{111} \widetilde{u}_{1}^{3}+\frac{1}{6} p_{222} \widetilde{v}_{1}^{3}+\frac{1}{6}\left(p_{112}+p_{121}+p_{211}\right) \widetilde{u}_{1}^{2} \widetilde{v}_{1}+\frac{1}{6}\left(p_{122}+p_{212}+p_{221}\right) \widetilde{u}_{1} \widetilde{v}_{1}^{2} \\
+p_{11} \widetilde{u}_{2} \widetilde{u}_{1}+p_{22} \widetilde{v}_{2} \widetilde{v}_{1}+\frac{1}{2}\left(p_{12}+p_{21}\right)\left(\widetilde{u}_{2} \widetilde{v}_{1}+\widetilde{u}_{1} \widetilde{v}_{2}\right) \\
\frac{1}{6} q_{111} \widetilde{u}_{1}^{3}+\frac{1}{6} q_{222} \widetilde{v}_{1}^{3}+\frac{1}{6}\left(q_{112}+q_{121}+q_{211}\right) \tilde{u}_{1}^{2} \widetilde{v}_{1}+\frac{1}{6}\left(q_{122}+q_{212}+q_{221}\right) \widetilde{u}_{1} \widetilde{v}_{1}^{2} \\
+q_{11} \widetilde{u}_{2} \widetilde{u}_{1}+q_{22} \widetilde{v}_{2} \widetilde{v}_{1}+\frac{1}{2}\left(q_{12}+q_{21}\right)\left(\widetilde{u}_{2} \widetilde{v}_{1}+\widetilde{u}_{1} \widetilde{v}_{2}\right)
\end{array}\right) .
\end{aligned}
$$

Meanwhile, the linear operator $L$ is dissembled by Taylor expansion and (14) transforms to

$$
L=L_{T}+\left(d_{1}^{T}-d_{1}\right) M
$$

where

$$
L_{T}=\left(\begin{array}{cc}
f_{\widetilde{u}}+d_{1}^{T} \nabla^{2} & f_{\widetilde{v}} \\
g_{\tilde{u}} & g_{\widetilde{v}}+d_{2} \nabla^{2}
\end{array}\right), M=\left(\begin{array}{cc}
-\nabla^{2} & 0 \\
0 & 0
\end{array}\right) .
$$

The derivation of the amplitude is

$$
\frac{\partial A}{\partial \widetilde{t}}=\varepsilon \frac{\partial A}{\partial T_{1}}+\varepsilon^{2} \frac{\partial A}{\partial T_{2}}+\ldots
$$

We substitute (17)-(21) into (14) and collect the $\mathcal{E}, \varepsilon^{2}$, and $\varepsilon^{3}$, represented by the following set of linear equations:

$$
\begin{aligned}
& \varepsilon: L_{T}\left(\begin{array}{c}
\tilde{u}_{1} \\
\tilde{v}_{1}
\end{array}\right)=0, \\
& \varepsilon^{2}: L_{T}\left(\begin{array}{c}
\tilde{u}_{2} \\
\tilde{v}_{2}
\end{array}\right)=\frac{\partial}{\partial T_{1}}\left(\begin{array}{c}
\tilde{u}_{1} \\
\tilde{v}_{1}
\end{array}\right)-d_{11} M\left(\begin{array}{c}
\tilde{u}_{1} \\
\tilde{v}_{1}
\end{array}\right)-\tilde{h}_{2} \triangleq\left(\begin{array}{c}
F_{\widetilde{u}} \\
F_{\tilde{v}}
\end{array}\right),
\end{aligned}
$$


TABLE 2: An example to illustrate the existence and stability of the equilibrium of model (4).

\begin{tabular}{ccccccc}
\hline$\eta$ & $\gamma$ & $e$ & $\tilde{u}^{*}$ & $\tilde{v}^{*}$ & $\operatorname{tr}(J)$ & $\operatorname{det}(J)$ \\
\hline 0.7 & 0.01040281036 & 140 & 0.351 & 33.74086284 & -0.3731305371 & 0.2532783223 \\
\hline
\end{tabular}

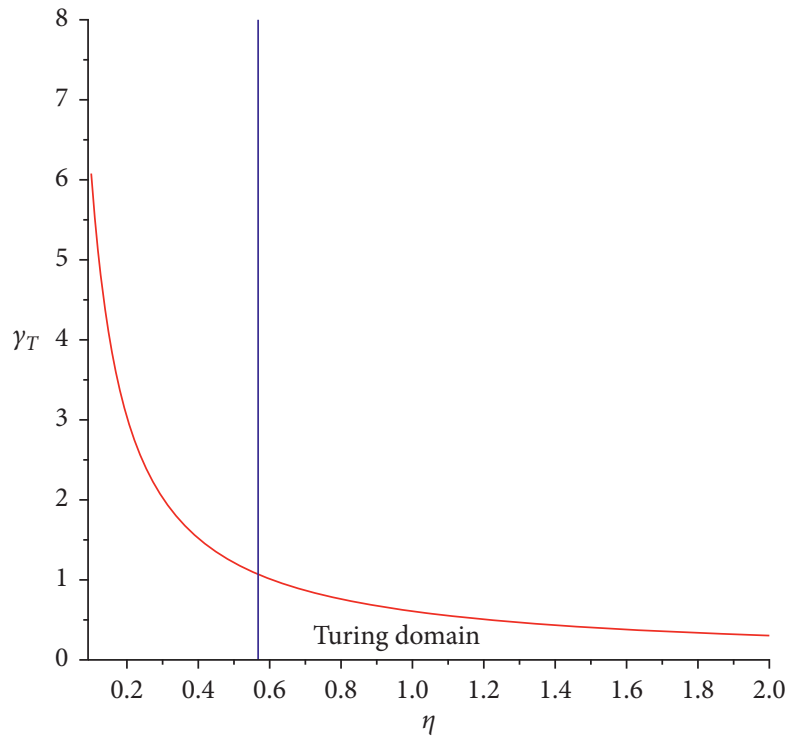

- Turing curve

- Hopf curve

Figure 1: Turing domain of model (4).

$$
\begin{aligned}
\varepsilon^{3}: L_{T}\left(\begin{array}{c}
\tilde{u}_{3} \\
\tilde{v}_{3}
\end{array}\right)= & \frac{\partial}{\partial T_{1}}\left(\begin{array}{c}
\tilde{u}_{2} \\
\tilde{v}_{2}
\end{array}\right)+\frac{\partial}{\partial T_{2}}\left(\begin{array}{c}
\tilde{u}_{1} \\
\tilde{v}_{1}
\end{array}\right)-d_{11} M\left(\begin{array}{c}
\tilde{u}_{2} \\
\tilde{v}_{2}
\end{array}\right) \\
& -d_{22} M\left(\begin{array}{c}
\tilde{u}_{1} \\
\tilde{v}_{1}
\end{array}\right)-\widetilde{h}_{3} \triangleq\left(\begin{array}{c}
H_{\tilde{u}} \\
H_{\tilde{v}}
\end{array}\right),
\end{aligned}
$$

where

$$
\left(\begin{array}{c}
F_{\widetilde{u}} \\
F_{\widetilde{v}}
\end{array}\right)=\sum_{j=1}^{3}\left(\begin{array}{c}
F_{\widetilde{u}}^{j} \\
F_{\widetilde{v}}^{j}
\end{array}\right) e^{i \mathbf{k}_{\mathbf{j}} \cdot \mathbf{r}}+\text { c.c. }
$$

and

$$
\left(\begin{array}{c}
H_{u} \\
H_{v}
\end{array}\right)=\sum_{j=1}^{3}\left(\begin{array}{c}
H_{u}^{j} \\
H_{v}^{j}
\end{array}\right) e^{i \mathbf{k}_{\mathbf{j}} \cdot \mathbf{r}}+\text { c.c.. }
$$

According to [43], solving (22), the solution is expressed

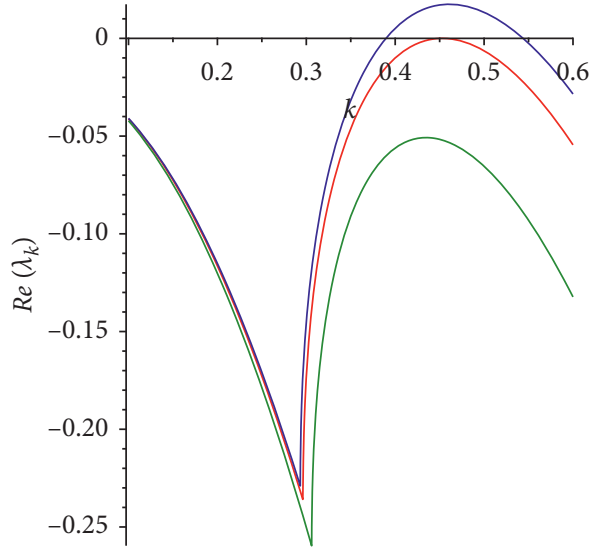

$$
\begin{aligned}
-d_{1} & =1.013052345 \\
-d_{1} & =0.95 \\
-d_{1} & =1.2
\end{aligned}
$$

FIgURE 2: Dispersion relation of system (4), which shows the real part of eigenvalues of model (4).

$$
\left(\begin{array}{c}
\tilde{u}_{1} \\
\tilde{v}_{1}
\end{array}\right)=\left(\begin{array}{l}
l \\
1
\end{array}\right)\left(W_{1} e^{i \mathbf{k}_{1} \cdot \mathbf{r}}+W_{2} e^{i \mathbf{k}_{2} \cdot \mathbf{r}}+W_{3} e^{i \mathbf{k}_{3} \cdot \mathbf{r}}\right)+\text { c.c. },
$$

where $l=f_{\widetilde{u}} d_{2}-g_{\widetilde{v}} d_{1}^{T} / 2 g_{\widetilde{u}} d_{1}^{T}$ and $\left|\mathbf{k}_{\mathbf{j}}\right|=k_{T}$. c.c. represents the complex conjugate and the pattern of the mode $\exp \left(i \mathbf{k}_{j} \cdot \mathbf{r}\right)$ is $W_{j}(j=1,2,3)$ under the first-order perturbation.

Substituting (27) into right-hand side of (23), the eigenvector of the operator $L_{T}^{+}$is as follows:

$$
\left(\begin{array}{l}
1 \\
l_{2}
\end{array}\right) e^{-i \mathbf{k}_{\mathrm{j}} \cdot \mathbf{r}}+\text { c.c., } \quad(j=1,2,3),
$$

where $L_{T}^{+}$represents the eigenvector of the zero eigenvalue of the adjoint operator of $L_{T}$ and

$$
l_{2}=\frac{2 f_{\widetilde{v}} d_{1}^{T}}{f_{\widetilde{u}} d_{2}-g_{\widetilde{v}} d_{1}^{T}}
$$

On the basis of Fredholm solvability condition, the orthogonality condition is given as as 


$$
\begin{aligned}
& \left(1, l_{2}\right)\left(\begin{array}{c}
F_{\tilde{u}}^{j} \\
F_{\tilde{v}}^{j}
\end{array}\right)=0, \quad(j=1,2,3) \\
& \left\{\begin{array}{l}
\left(l+l_{2}\right) \frac{\partial W_{1}}{\partial T_{1}}=d_{11} k_{T}^{2} l W_{1}+\left[p_{11} l^{2}+\left(p_{12}+p_{21}\right) l+p_{22}+l_{2}\left(q_{11} l^{2}+\left(q_{12}+q_{12}\right) l+q_{22}\right] \bar{W}_{2} \bar{W}_{3}\right. \\
\left(l+l_{2}\right) \frac{\partial W_{2}}{\partial T_{1}}=d_{11} k_{T}^{2} l W_{2}+\left[p_{11} l^{2}+\left(p_{12}+p_{21}\right) l+p_{22}+l_{2}\left(q_{11} l^{2}+\left(q_{12}+q_{12}\right) l+q_{22}\right)\right] \bar{W}_{1} \bar{W}_{3} \\
\left(l+l_{2}\right) \frac{\partial W_{3}}{\partial T_{1}}=d_{11} k_{T}^{2} l W_{3}+\left[p_{11} l^{2}+\left(p_{12}+p_{21}\right) l+p_{22}+l_{2}\left(q_{11} l^{2}+\left(q_{12}+q_{12}\right) l+q_{22}\right)\right] \bar{W}_{1} \bar{W}_{2}
\end{array}\right.
\end{aligned}
$$

Let the solution of (23) have the following form:

$$
\begin{aligned}
\left(\begin{array}{c}
\tilde{u}_{2} \\
\tilde{v}_{2}
\end{array}\right)= & \left(\begin{array}{c}
\tilde{U}_{0} \\
\tilde{V}_{0}
\end{array}\right)+\sum_{j=1}^{3}\left(\begin{array}{c}
\tilde{U}_{j} \\
\tilde{V}_{j}
\end{array}\right) e^{i \mathbf{k}_{j} \cdot \mathbf{r}}+\sum_{j=1}^{3}\left(\begin{array}{c}
\tilde{U}_{j j} \\
\tilde{V}_{j j}
\end{array}\right) e^{i 2 \mathbf{k}_{j} \mathbf{r}} \\
& +\left(\begin{array}{c}
\tilde{U}_{12} \\
\tilde{V}_{12}
\end{array}\right) e^{i\left(\mathbf{k}_{1}-\mathbf{k}_{2}\right) \mathbf{r}} \\
& +\left(\begin{array}{c}
\tilde{U}_{23} \\
\tilde{V}_{23}
\end{array}\right) e^{i\left(\mathbf{k}_{2}-\mathbf{k}_{3}\right) \cdot \mathbf{r}}+\left(\begin{array}{c}
\tilde{U}_{31} \\
\tilde{V}_{31}
\end{array}\right) e^{i\left(\mathbf{k}_{3}-\mathbf{k}_{1}\right) \cdot \mathbf{r}}+\text { c.c.. }
\end{aligned}
$$

$$
\begin{aligned}
\left(\begin{array}{c}
\tilde{U}_{0} \\
\tilde{V}_{0}
\end{array}\right) & =\left(\begin{array}{c}
\tilde{u}_{0} \\
\tilde{v}_{0}
\end{array}\right)\left(\left|W_{1}\right|^{2}+\left|W_{2}\right|^{2}+\left|W_{3}\right|^{2}\right), \\
\tilde{U}_{j} & =l \widetilde{V}_{j}, \\
\left(\begin{array}{c}
\tilde{U}_{i j} \\
\tilde{V}_{i j}
\end{array}\right) & =\left(\begin{array}{c}
\tilde{u}_{11} \\
\tilde{v}_{11}
\end{array}\right) W_{j}^{2}, \\
\left(\begin{array}{c}
\tilde{U}_{m n} \\
\tilde{V}_{m n}
\end{array}\right) & =\left(\begin{array}{c}
\tilde{u}_{m n} \\
\tilde{v}_{m n}
\end{array}\right) W_{m} \bar{W}_{n},
\end{aligned}
$$

where

Substituting (32) into (23) and merging the coefficients of $\exp (0), \exp \left(i \mathbf{k}_{j} \cdot \mathbf{r}\right), \exp \left(i 2 \mathbf{k}_{j} \cdot \mathbf{r}\right)$, and $\exp \left(i\left(\mathbf{k}_{j}-\mathbf{k}_{i}\right) \cdot \mathbf{r}\right)$, we obtain the following resultant relation:

$$
\begin{aligned}
& \left(\begin{array}{c}
\widetilde{u}_{0} \\
\widetilde{v}_{0}
\end{array}\right)=\frac{1}{f_{\widetilde{u} g_{\tilde{v}}-f_{\widetilde{v}} g_{\tilde{u}}}}\left(\begin{array}{c}
-g_{\tilde{v}}\left[p_{11} l^{2}+\left(p_{12}+p_{21}\right) l+p_{22}\right]+p_{\tilde{v}}\left[q_{11} l^{2}+\left(q_{12}+q_{12}\right) l+q_{22}\right] \\
-f_{\widetilde{u}}\left[q_{11} l^{2}+\left(q_{12}+q_{12}\right) l+q_{22}\right]+q_{\tilde{u}}\left[p_{11} l^{2}+\left(p_{12}+p_{21}\right) l+p_{22}\right]
\end{array}\right) \\
& \left(\begin{array}{c}
\tilde{u}_{11} \\
\widetilde{v}_{11}
\end{array}\right)=\frac{1}{2} \frac{1}{\left(f_{\tilde{u}}-4 K_{T}^{2} d_{1}^{T}\right)\left(g_{\tilde{v}}-4 K_{T}^{2} d_{2}\right)-f_{\tilde{v}} g_{\tilde{u}}}\left(\begin{array}{c}
-\left(g_{\tilde{v}}-4 K_{T}^{2} d_{2}\right)\left[p_{11} l^{2}+\left(p_{12}+p_{21}\right) l+p_{22}\right]+p_{\tilde{v}}\left[q_{11} l^{2}+\left(q_{12}+q_{12}\right) l+q_{22}\right] \\
-\left(f_{\tilde{u}}-4 K_{T}^{2} d_{1}^{T}\right)\left[q_{11} l^{2}+\left(q_{12}+q_{12}\right) l+q_{22}\right]+g_{\tilde{u}}\left[f_{11} l^{2}+\left(p_{12}+p_{21}\right) l+p_{22}\right]
\end{array}\right), \\
& \left(\begin{array}{c}
\tilde{u}_{m n} \\
\tilde{v}_{m n}
\end{array}\right)=\frac{1}{\left(f_{\widetilde{u}}-3 K_{T}^{2} d_{1}^{T}\right)\left(g_{\widetilde{v}}-3 K_{T}^{2} d_{22}\right)-f_{\widetilde{v}} g_{\widetilde{u}}}\left(\begin{array}{c}
-\left(g_{\widetilde{v}}-3 K_{T}^{2} d_{2}\right)\left[p_{11} l^{2}+\left(p_{12}+p_{21}\right) l+p_{22}\right]+f_{\widetilde{v}}\left[q_{11} l^{2}+\left(q_{12}+q_{12}\right) l+q_{22}\right] \\
-\left(f_{\widetilde{u}}-3 K_{T}^{2} a d d_{1}^{T}\right)\left[q_{11} l^{2}+\left(q_{12}+q_{12}\right) l+q_{22}\right]+q_{\tilde{u}}\left[p_{11} l^{2}+\left(p_{12}+p_{21}\right) l+p_{22}\right]
\end{array}\right) .
\end{aligned}
$$

We make use of the Fredholm solvability condition in (24). Now, we have

$$
\left(1, l_{2}\right)\left(\begin{array}{c}
H_{\tilde{u}}^{j} \\
H_{\tilde{v}}^{j}
\end{array}\right)=0 \quad(j=1,2,3)
$$


From (35), we get

$$
\left\{\begin{array}{l}
\left(l+l_{2}\right)\left(\frac{\partial V_{1}}{\partial T_{1}}+\frac{\partial W_{1}}{\partial T_{2}}\right)=l k_{T}^{2}\left(d_{11} V_{1}+d_{22} W_{1}\right)+D\left(\bar{V}_{2} \bar{W}_{3}+\bar{V}_{3} \bar{W}_{2}\right)+\left(E_{1}\left|W_{1}\right|^{2}+E_{2}\left(\left|W_{2}\right|^{2}+\left|W_{3}\right|^{2}\right)\right) W_{1}, \\
\left(l+l_{2}\right)\left(\frac{\partial V_{2}}{\partial T_{1}}+\frac{\partial W_{2}}{\partial T_{2}}\right)=l k_{T}^{2}\left(d_{11} V_{2}+d_{22} W_{2}\right)+D\left(\bar{V}_{1} \bar{W}_{3}+\bar{V}_{3} \bar{W}_{1}\right)+\left(E_{1}\left|W_{2}\right|^{2}+E_{2}\left(\left|W_{1}\right|^{2}+\left|W_{3}\right|^{2}\right)\right) W_{2}, \\
\left(l+l_{2}\right)\left(\frac{\partial V_{3}}{\partial T_{1}}+\frac{\partial W_{3}}{\partial T_{2}}\right)=l k_{T}^{2}\left(d_{11} V_{3}+d_{22} W_{3}\right)+D\left(\bar{V}_{1} \bar{W}_{2}+\bar{V}_{2} \bar{W}_{1}\right)+\left(E_{1}\left|W_{3}\right|^{2}+E_{2}\left(\left.W_{1}\right|^{2}+\left|W_{2}\right|^{2}\right)\right) W_{3},
\end{array}\right.
$$

where

$$
\begin{aligned}
& D=\left(l r_{1}+s_{1}\right)+l_{2}\left(l r_{2}+n_{2}\right), \\
& E_{1}=\left(b r_{1}+a_{1} s_{1}+\frac{P_{1}}{2}\right)+l_{2}\left(b r_{2}+b_{1} s_{2}+\frac{P_{2}}{2}\right), \\
& E_{2}=\left(c r_{1}+c_{1} s_{1}+P_{1}\right)+l_{2}\left(c r_{2}+c_{1} s_{2}+P_{2}\right),
\end{aligned}
$$

and

$$
\begin{aligned}
b= & \widetilde{u}_{0}+\widetilde{u}_{11}, c=\widetilde{u}_{0}+\widetilde{u}_{m n}, \\
b_{1}= & \widetilde{v}_{0}+\widetilde{v}_{11}, c_{1}=\widetilde{v}_{0}+\widetilde{v}_{m n}, \\
r_{1}= & p_{11} l+\frac{1}{2}\left(p_{12}+p_{21}\right), s_{1}=p_{22}+\frac{1}{2}\left(p_{12}+p_{21}\right) l, \\
r_{2}= & q_{11} l+\frac{1}{2}\left(q_{12}+q_{21}\right), s_{2}=q_{22}+\frac{1}{2}\left(q_{12}+q_{21}\right) l, \\
P_{1}= & p_{111} l^{3}+\left(p_{112}+p_{121}+p_{211}\right) l^{2} \\
& +\left(p_{122}+p_{212}+p_{221}\right) l+p_{222}, \\
P_{2}= & q_{111} l^{3}+\left(q_{112}+q_{121}+q_{211}\right) l^{2} \\
& +\left(q_{122}+q_{212}+q_{221}\right) l+q_{222} .
\end{aligned}
$$

Multiplying (31) and (36) by $\varepsilon$ and $\varepsilon^{2}$, together with (21) and

$$
D_{i}=D_{i}^{\tilde{u}}=l D_{i}^{\tilde{v}}, \quad(i=1,2,3),
$$

we have the following amplitude equations:

$$
\left\{\begin{array}{l}
\tau_{0} \frac{\partial A_{1}}{\partial t}=\mu D_{1}+h \bar{D}_{2} \bar{D}_{3}-\left(g_{1}\left|D_{1}\right|^{2}+g_{2}\left(\left|D_{2}\right|^{2}+\left|D_{3}\right|^{2}\right)\right) D_{1}, \\
\tau_{0} \frac{\partial A_{2}}{\partial t}=\mu D_{2}+h \bar{D}_{1} \bar{D}_{3}-\left(g_{1}\left|D_{2}\right|^{2}+g_{2}\left(\left|D_{1}\right|^{2}+\left|D_{3}\right|^{2}\right)\right) D_{2} \\
\tau_{0} \frac{\partial A_{3}}{\partial t}=\mu D_{3}+h \bar{D}_{1} \bar{D}_{2}-\left(g_{1}\left|D_{3}\right|^{2}+g_{2}\left(\left|D_{1}\right|^{2}+\left|D_{2}\right|^{2}\right)\right) D_{3}
\end{array}\right.
$$

where

$$
\begin{gathered}
\tau_{0}=\frac{l+l_{2}}{d_{1}^{T} l k_{T}^{2}}, \mu=\frac{d_{1}^{T}-d_{1}}{d_{1}^{T}}, h=\frac{D}{d_{1}^{T} l^{2} k_{T}^{2}}, g_{1} \\
=-\frac{E_{1}}{d_{1}^{T} l^{3} k_{T}^{2}}, g_{2}=-\frac{E_{2}}{d_{1}^{T} l^{3} k_{T}^{2}} .
\end{gathered}
$$

On the basis of aforementioned analysis, we obtain the following results about the pattern selection of system (4) shown in (Table 3 ).

\section{Numerical Simulations of Patterns}

4.1. Pattern Selection. In this section, we select the suitable value of $d_{1}$ and perform numerical simulations to verify the theoretical results, i.e., to see whether the patterns arise in the corresponding Turing domain. We make use of the zeroflux boundary condition with $200 \times 200$ lattice sites. The space step is $\Delta h=1$, and the time step is $\Delta t=0.01$. For the sake of the similar type of patterns of the predator and prey, we choose the predator to exhibit our investigations. We start with initial spatial state as the heterogeneity space. The simulations are performed until the patterns do not change their characteristic anymore. The value of parameters is presented in Tables 4 and 5 .

Figure 3 shows the evolutions of spatial pattern of the predator at final instants when $d_{1}=0.95, d_{1}=0.5, d_{1}=0.47$, and $d_{1}=0.2$, respectively. As presented in Figure 3 , we obtain three different patterns in the corresponding Turing domains, which are hot spot patterns, i.e., Figure 3(a), mixture of spots and stripes, i.e., Figures $3(\mathrm{~b})$ and $3(\mathrm{c})$, and stripe patterns, i.e., Figure 3(d). Thus, the simulation results shown are aligned with the theoretical analysis. However, it is noticeable that there is a little difference between the two mixtures in Figure 3(c) and in Figure 3(d), because they have different wavelengths.

4.2. Effect of the Prey's Diffusion on Patterns of Predator. In this section, we discuss the pattern formation driven by the diffusion rate of the prey by varying the value of the prey's diffusion, while the values of the other parameters are kept the same as in Section 4.1. 
TABLE 3: The pattern selection of model (4).

\begin{tabular}{lcc}
\hline$\mu$ & Pattern & Stability \\
$\mu_{2}<\mu=0.1115957587<\mu_{3}$ & Spot pattern & Stable \\
$\mu_{3}<\mu=0.5064420882<\mu_{4}$ & Coexistence of the spots and stripe patterns & Bistable \\
$\mu=0.8025768353>\mu_{4}$ & Tripe patterns & Stable \\
\hline
\end{tabular}

TABLe 4: The parameters set in system (4).

\begin{tabular}{lccc}
\hline$\eta$ & $\gamma$ & $e$ & $d_{2}$ \\
0.6 & 0.002056779432 & 525 & 4 \\
\hline
\end{tabular}

TABLE 5: The parameters calculated in system (4).

\begin{tabular}{lccccccc}
\hline$d_{1}^{T}$ & $g_{1}$ & $g_{2}$ & $h$ & $\mu_{1}$ & $\mu_{2}$ & $\mu_{3}$ & $\mu_{4}$ \\
1.013052345 & 76.6130004 & 171.1217394 & 4.675460751 & -0.01304738873 & 0 & 0.1875026305 & 0.7938085466 \\
\hline
\end{tabular}

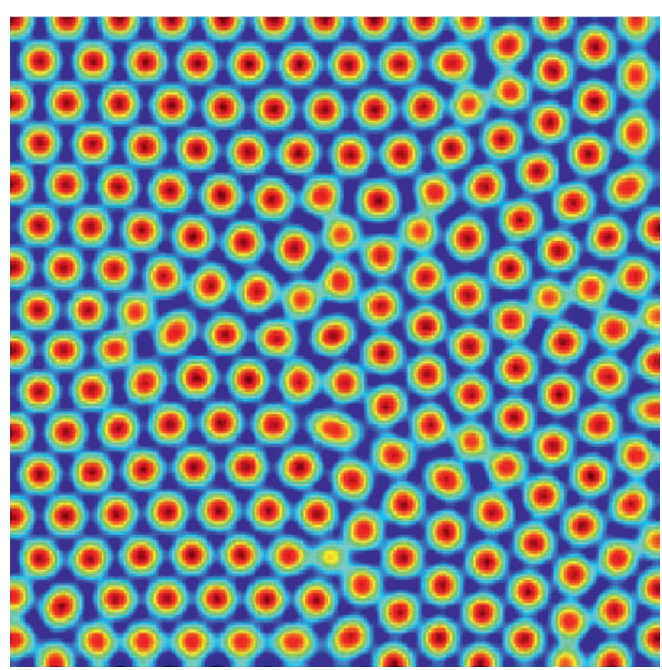

(a)

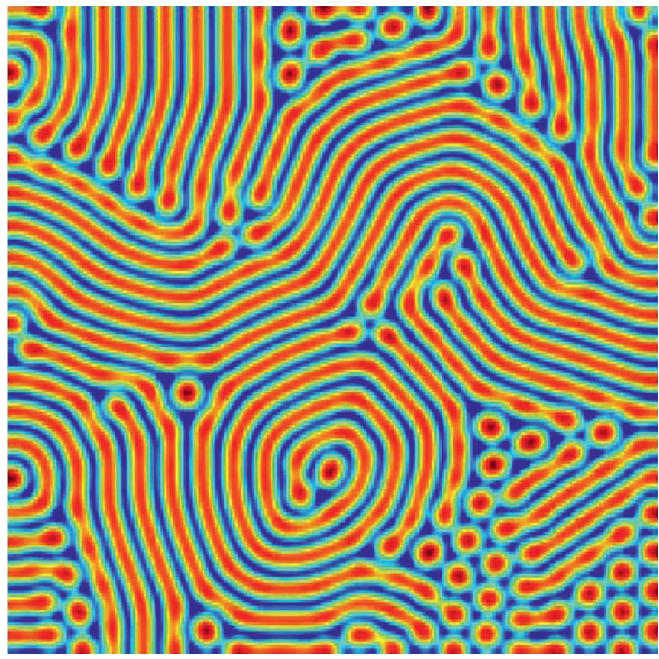

(c)
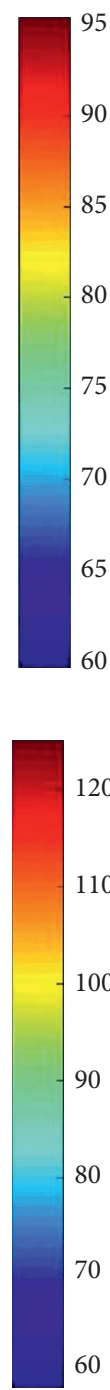

60

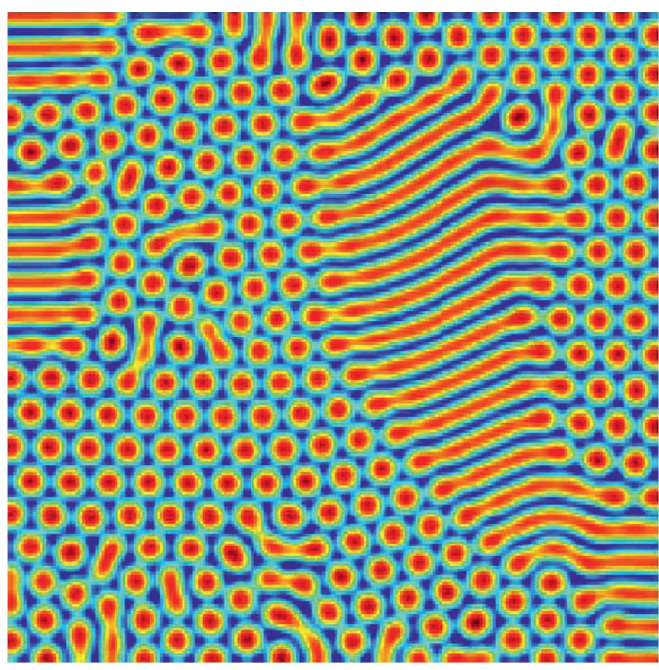

(b)

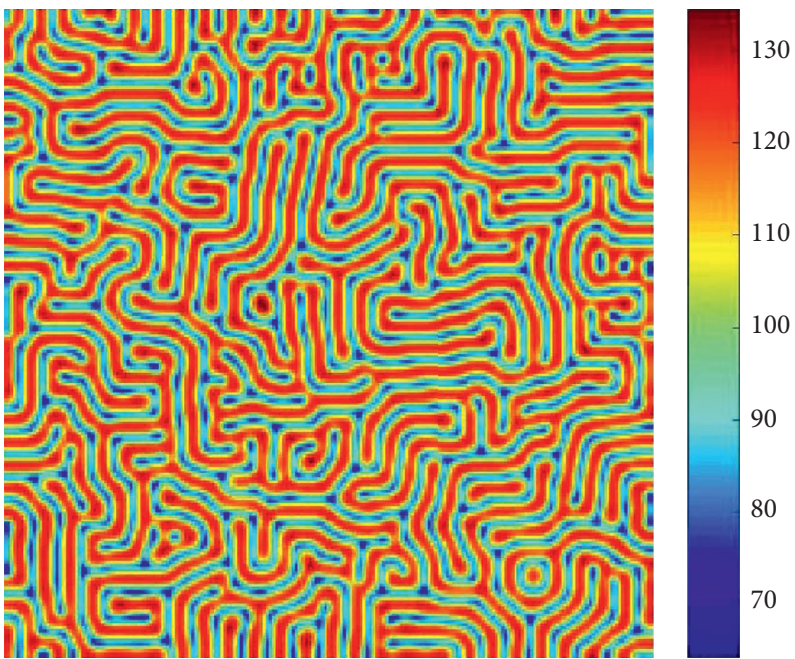

(d)

Figure 3: The spatial patterns of the predator with different values of 31 : (a) $31=0.95$; (b) $31=0.5$; (c) $31=0.47$; (d) $31=0.2$. 

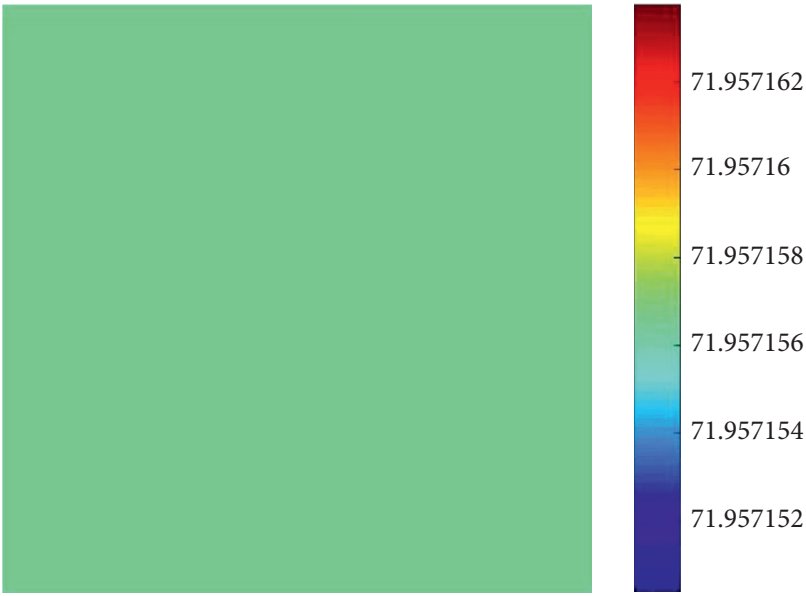

(a)


(c)

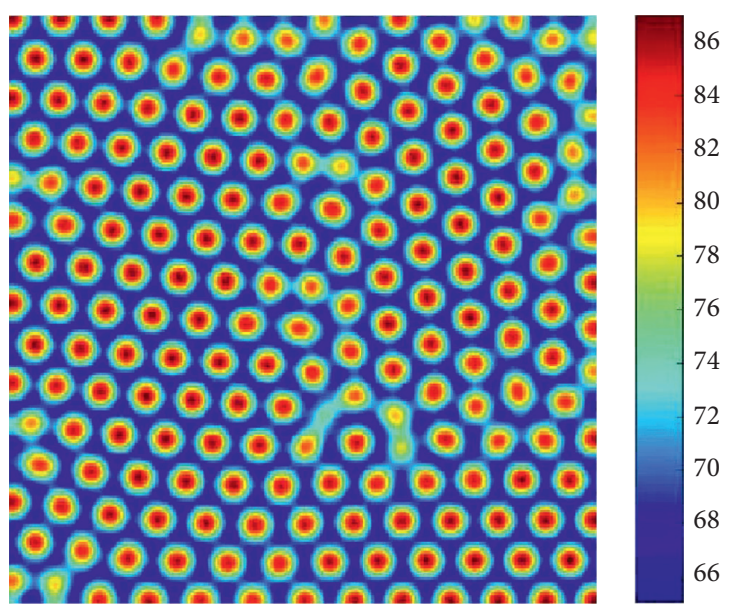

(b)

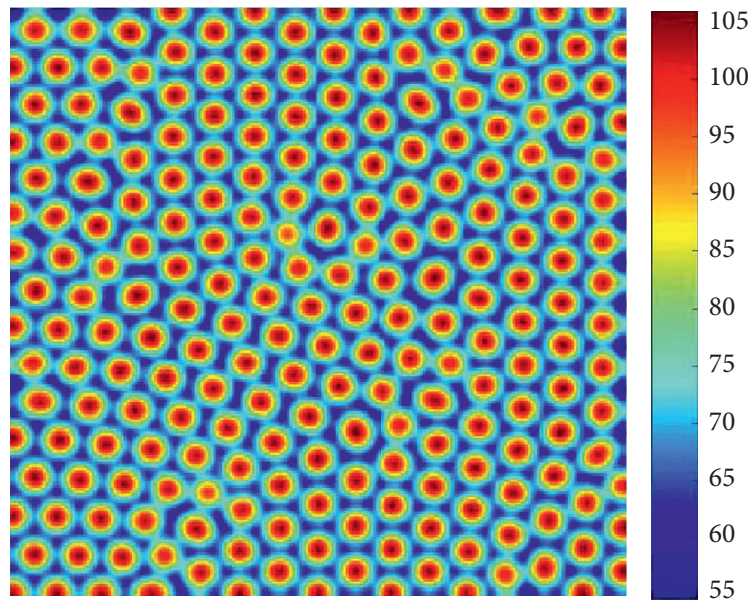

(d)

FIgURE 4: The spatial patterns of the predator with different value of 31 : (a) $31=1.025$; (b) $31=1.009$; (c) $31=0.9$; (d) $31=0.83$.

First, we fix $d_{1}=1.025$ which is larger than $d_{1}^{T}$ and transcends the bifurcation domain. Please note that there are no patterns emerging in Figure 4(a). This is in line with our theoretical results. As $d_{1}$ becomes smaller than $d_{1}^{T}$ decreasing from 1.009 to 0.83 in Figures 4(b)-4(d), the spot pattern gets more and more dense. In order to analyze the variation in density, we compare Figure 4(b) with Figure 4(d) at the same iterations in detail. On the one hand, from Figures 5 and 6, we find that Figure 6 transforms faster than Figure 5. For example, Figure 6(b) starts to become the spot pattern while Figure 5(b) is still irregular in shape after 3400 iterations. On the contrary, we observe that the spots in Figure 5(d) are sparser than in Figure 6(d). Thus, the density of the predator will decrease as there is an increase in the mobility of the prey. According to the Reichenbach's viewpoint [44], we can illustrate our results effectively, i.e., when the prey species move frequently, the prey will encounter the predator easily. This leads to the decrease of the prey and aggravate the competition of the predator. As a result, the predators confront the danger of extinction. Moreover, we conclude that the pattern formation is induced by the diffusion rate of the prey by comparing Figure 4(a) with Figures 4(b)-4(d).
4.3. Pattern Transitions of the Predator Driven by Parameter $\gamma$. We fit the values of parameters $\eta, e, d_{1}$, and $d_{2}$ presented in Table 6 , while the value of $\gamma$ gradually increases. We investigate the changes in the spatial distribution of the predator as the conversion rate into predator biomass varies. Specifically, when $\gamma$ is fixed $\gamma=0.007041633659$, hot spot pattern dominates the spatial region in Figure 7(a). In Figure 7(b), when we have $\gamma=0.008509501103$, then the mixture of hot spots and stripes appears. As $\gamma$ continues to grow to 0.009332994621 , there is just the stripe pattern that is presented in Figure 5(c). Similarly, when $\gamma$ increases to $\gamma=0.01032204568$, the mixture of cold spots and stripes arises, which is shown in Figure 7(d). Finally, when $\gamma$ becomes larger, we simulate the cold spot pattern presented in Figure 7(e).Figure 7 shows the orderly changes of the pattern transition, as well as the density of the predator increases from 85 to 90 and then decreases to 85 again with an increase in the value of $\gamma$, because $\gamma$ denotes the number of the prey species which are captured by a single predator. The higher the value of parameter $\gamma$, the more food the predator species eat. However, please note that there is an effect of the environmental capacity, so the number of the predators cannot 

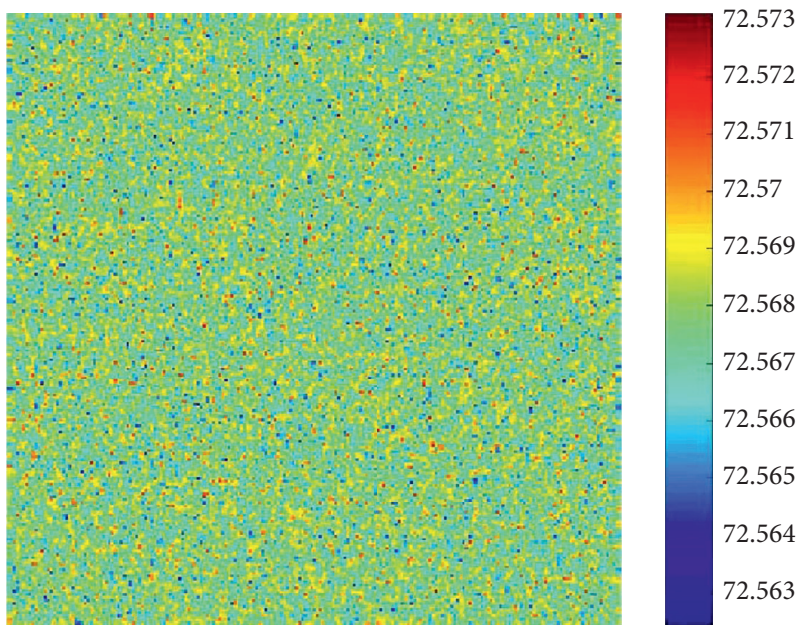

(a)

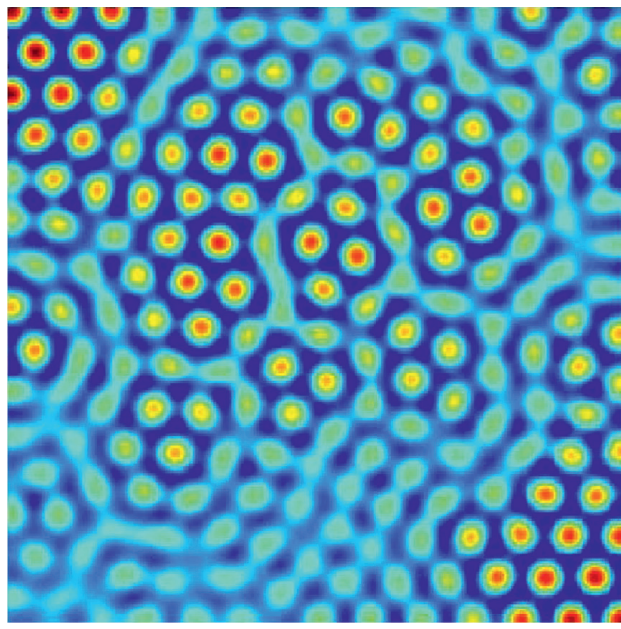

(c)

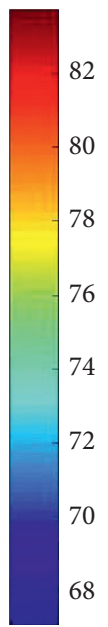

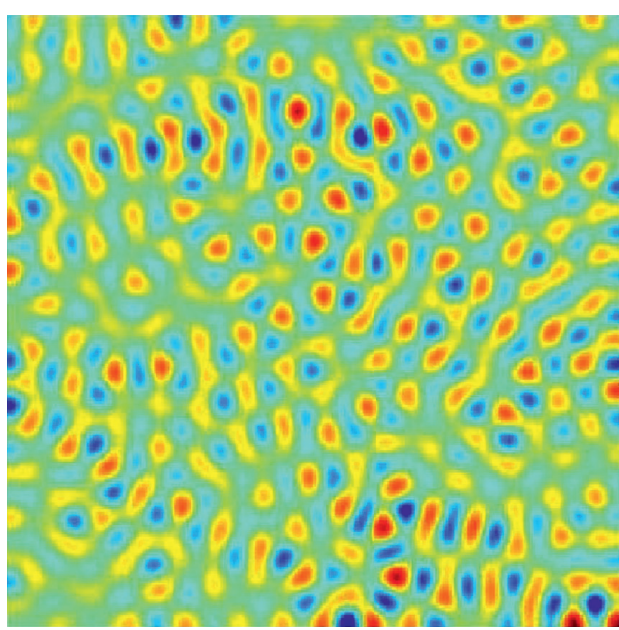

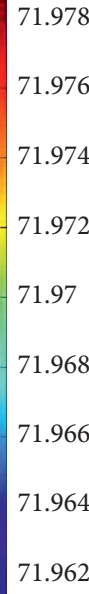

(b)

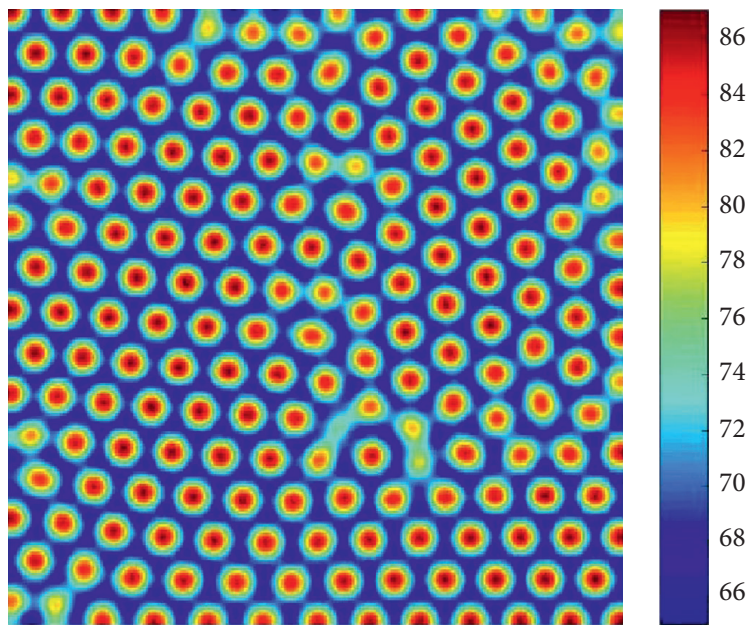

(d)

FIGURE 5: The iterations of stripe patterns of the predator with $31=1.009$. The number of iterations is (a) 0 , (b) 3600 , (c) 130000 , and (d) 150000 .

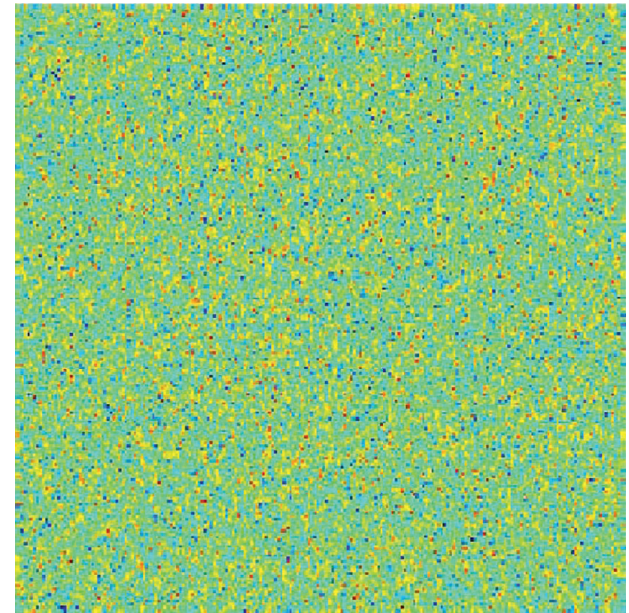

(a)
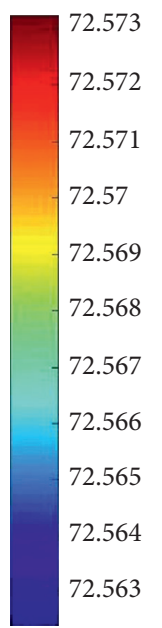

72.563

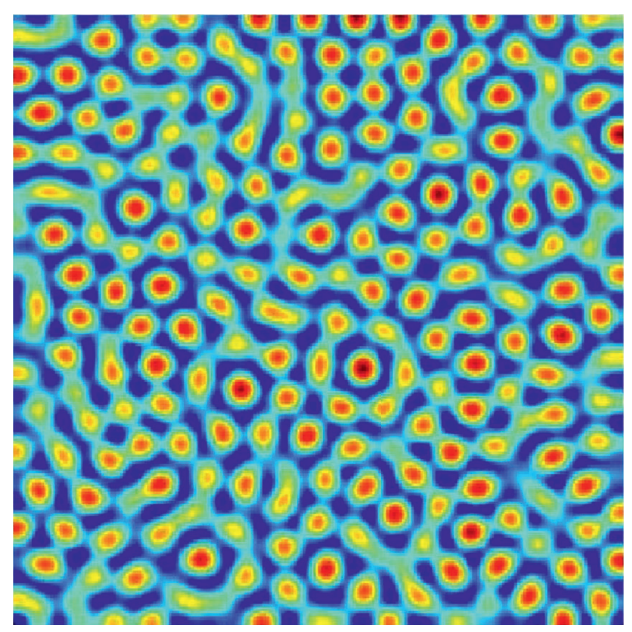

(b)

FIGURE 6: Continued.

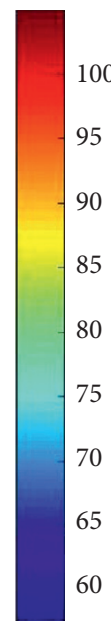

. 


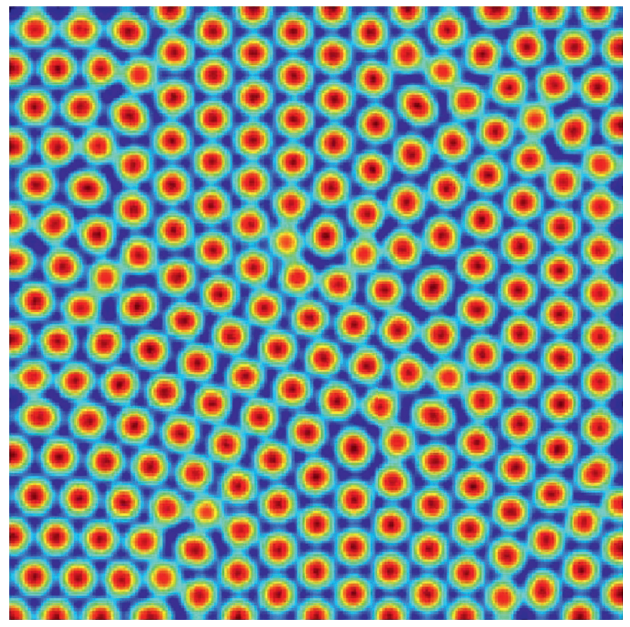

(c)

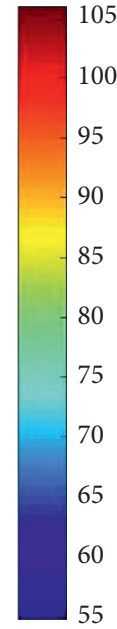

Figure 6: The iterations of stripe patterns of the predator with $31=0.83$. The number of iterations is (a) 0 , (b) 3600 , (c) 130000 , and (d) 150000 .

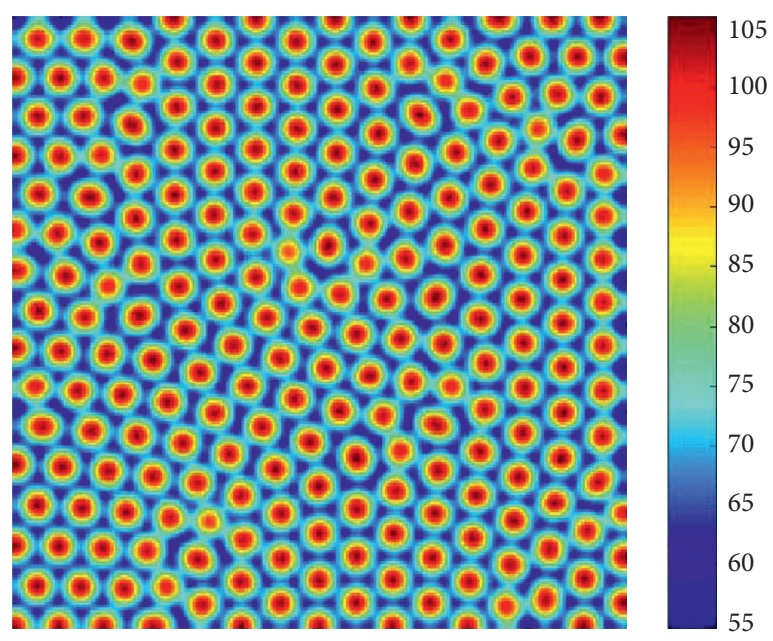

(d)

TABLE 6: The parameters selected in system (4).

\begin{tabular}{lccc}
\hline$\eta$ & $e$ & $d_{1}$ & \\
0.6 & 350 & $d_{2}$ & 0.2 \\
\hline
\end{tabular}

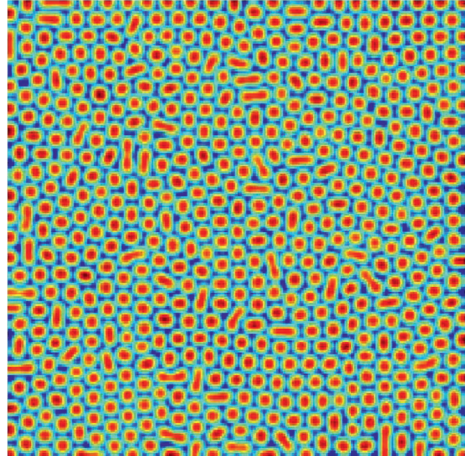

(a)

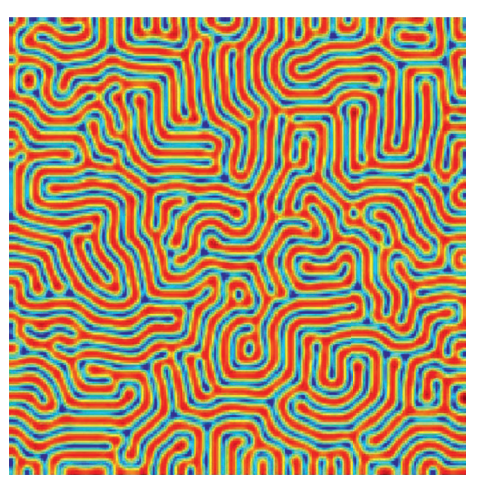

(c)
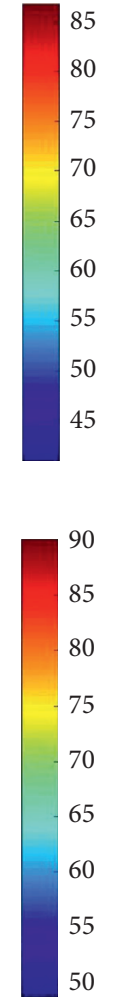

11ก:0

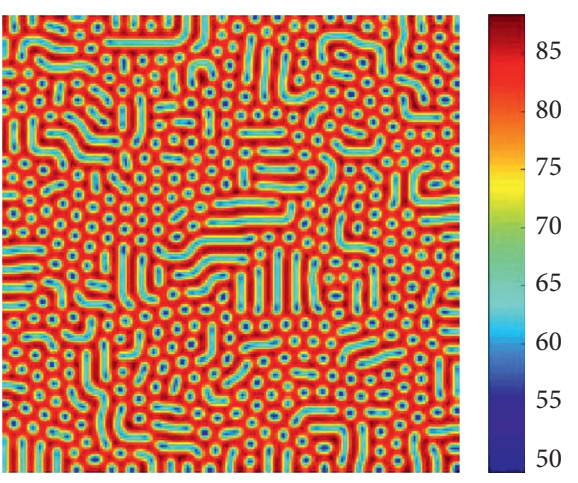

FIgURE 7: Continued.

(d)

- 


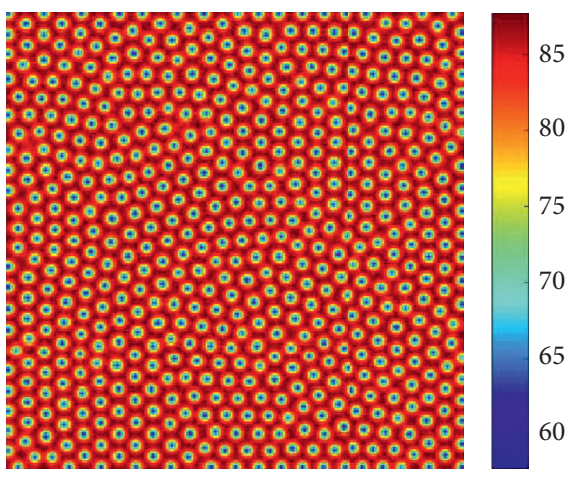

(e)

FigURE 7: The transitions patterns of the predator induced by the conversion rate of the prey into predator biomass and the other parameters are set in Table 6. Iterations: (a) 0.007041633659; (b) 0.008509501103; (c) 0.009332994621; (d) 0.01032204568; (e) 0.01092704597.

increase forever. So, the population of the predator decreases again when it exceeds the threshold value. The simulation results of this work present a clear biological meaning.

\section{Conclusions}

In this work, we study a ratio-dependent prey-predator model. The pattern selection of our model has been investigated via multiple scale analysis near the Turing bifurcation point. First, we obtain the condition of the Turing instability of our model. Then, by choosing $d_{1}$ as a bifurcation parameter, we obtained the amplitude equations and presented the Turing pattern selection of model (4). The simulation results presented in this work are aligned with the theoretical analysis, i.e., $H_{0}$ spot patterns arise when $\mu$ lies in domain $\left(\mu_{2}, \mu_{3}\right)$ with $d_{1}=0.95$; the stable mixture of spots and stripes appears when $\mu_{3}<\mu<\mu_{4}$ with $d_{1}=0.5$ and $d_{1}=0.47$, while only stripe pattern is simulated when $\mu>\mu_{4}$ and $d_{1}=0.2$. On the contrary, we study the effect of the spatial motion and conversion rate of predator biomass on density of the predator. The decreasing value of the former agent $d_{1}$ causes the sparse density in the predator population, while the increasing value of the latter factor induces the variation of the density of predator species. The simulation results presented in this work reveal the inherent mechanism of our model. Moreover, on the basis of our analysis, the slight value changes in $d_{1}$ lead to density changes of the predator. This observation shows that the diffusive rate of the prey has a great effect on prey and the whole system. Above all, the intrinsic agents, i.e., diffusion and predation, control the persistence or extinction of the spatial predator-prey model [45].

However, it is noticeable that there is a little defect in the analysis of pattern selection via amplitude equations. That is, pattern selection is only valid when the value of the controlled parameter $d_{1}$ is set close to the threshold $d_{1}^{T}$. Thus, pattern selection is only valid when the value is very close to the controlled parameter $d_{1}^{T}$. This inspires us to think about the best distance of the parameter away from the controlled parameter. Besides, the method of the amplitude equations is also applicable in the epidemic model and the vegetation model to study the pattern formation of the epidemic and vegetation in the future [46-49].

\section{Data Availability}

The data in the manuscript are not the public data.

\section{Conflicts of Interest}

The authors declare that they have no conflicts of interest.

\section{Acknowledgments}

This work was supported by the Youth Science and Technology Research Foundation of Shanxi Province (Grant No. 201801D221033), Universities' Science and Technology Innovation Item of Shanxi Province (Grant No. 2019L0472), the Key Construction Disciplines Project of Xinzhou Teachers University (XK201501), and Scientific Research Project of Xinzhou Teachers University (2019KY07).

\section{References}

[1] P. H. Leslie, "Some further notes on the use of matrices in population mathematics," Biometrika, vol. 35, no. 3-4, pp. $213-245,1948$.

[2] P. H. Leslie and J. C. Gower, "The properties of a stochastic model for the predator-prey type of interaction between two species," Biometrika, vol. 47, no. 3-4, pp. 219-234, 1960.

[3] E. Sáez and E. González-Olivares, "Dynamics of a predatorprey model," Siam Journal on Applied Mathematics, vol. 59, no. 5, pp. 1867-1878, 1999.

[4] Z.-P. Ma and W.-T. Li, "Bifurcation analysis on a diffusive Holling-Tanner predator-prey model," Applied Mathematical Modelling, vol. 37, no. 6, pp. 4371-4384, 2013.

[5] X. Li, W. Jiang, and J. Shi, "Hopf bifurcation and Turing instability in the reaction-diffusion Holling-Tanner predatorprey model," IMA Journal of Applied Mathematics, vol. 78, no. 2, pp. 287-306, 2013.

[6] R. M. May, "Limit cycles in predator-prey communities," Science, vol. 177, no. 4052, pp. 900-902, 1972.

[7] G.-Q. Sun, J. Zhang, L.-P. Song, Z. Jin, and B.-L. Li, "Pattern formation of a spatial predator-prey system," Applied 
Mathematics and Computation, vol. 218, no. 22, pp. 1115111162, 2012.

[8] P. S. Mandal and M. Banerjee, "Stochastic persistence and stationary distribution in a Holling-Tanner type prey-predator model," Physica A, vol. 391, no. 4, pp. 1216-1233, 2012.

[9] Z. Lu and X. Liu, "Analysis of a predator-prey model with modified Holling-Tanner functional response and time delay," Nonlinear Analysis: Real World Applications, vol. 9, no. 2, pp. 641-650, 2008.

[10] W. Zuo and D. Jiang, "Periodic solutions for a stochastic non-autonomous Holling-Tanner predator-prey system with impulses," Nonlinear Analysis: Hybrid Systems, vol. 22, pp. 191-201, 2016.

[11] B. Wang, A.-L. Wang, Y.-J. Liu, and Z.-H. Liu, "Analysis of a spatial predator-prey model with delay," Nonlinear Dynamics, vol. 62, no. 3, pp. 601-608, 2010.

[12] P. S. Mandal and M. Banerjee, "Stochastic persistence and stability analysis of a modified Holling-Tanner model," Mathematical Methods in The Applied Sciences, vol. 36, no. 10, pp. 1263-1280, 2013.

[13] L. Chang, G.-Q. Sun, Z. Wang, and Z. Jin, "Rich dynamics in a spatial predator-prey model with delay," Applied Mathematics and Computation, vol. 256, pp. 540-550, 2015.

[14] M. S. Peixoto, L. C. de Barros, and R. C. Bassanezi, "Predator-prey fuzzy model," Ecologicall Modelling, vol. 214, no. 1, pp. 39-44, 2008.

[15] M. S. Peixoto, L. C. Barros, and R. C. Bassanezi, "An approach via fuzzy sets theory for predator-prey model," Decision Making and Soft Computing, vol. 9, pp. 682-687, 2014.

[16] C. S. Holling, "The functional response of predators to prey density it's role in mimicry and population regulation," Memoirs of the Entomological Society of Canada, vol. 97, no. 45, pp. 5-60, 1965.

[17] Y. Huang, F. Chen, and L. Zhong, "Stability analysis of a prey-predator model with holling type III response function incorporating a prey refuge," Applied Mathematics and Computation, vol. 182, no. 1, pp. 672-683, 2006.

[18] J. H. P. Dawes and M. O. Souza, "A derivation of Holling's type I, II and III functional responses in predator-prey systems," Journal of Theoretical Biology, vol. 327, pp. 11-22, 2013.

[19] L. A. Real, "Ecological determinants of functional response," Ecology, vol. 60, no. 3, pp. 481-485, 1979.

[20] M. Rietkerk, M. C. Boerlijst, and F. van Langevelde, "Self-organization of vegetation in arid ecosystems," Journal of Ecology, vol. 94, pp. 537-547, 2002.

[21] M. Rietkerk, S. C. Dekker, and P. C. de Ruiter, "Self-organized patchiness and catastrophic shifts in ecosystems," Science, vol. 305, no. 5692, pp. 1926-1929, 2004.

[22] M. Rietkerk and J. van de Koppel, "Regular pattern formation in real ecosystems," Trends in Ecology \& Evolution, vol. 23, no. 3, pp. 169-175, 2008.

[23] A. Turing, "The chemical basis of morphogenesis," Bulletin of Mathematical Biology, vol. 52, no. 1-2, pp. 153-197, 1990.

[24] H. Meinhardt, "Pattern formation in biological systems," Reference Module in Materials Science and Materials Engineering, Elsevier, Amsterdam, Netherlands, 2016.

[25] V. K. Vanag and I. R. Epstein, "Cross-diffusion and pattern formation in reaction-diffusion systems," Physical Chemistry Chemical Physics, vol. 11, no. 6, pp. 897-912, 2009.

[26] Y. Almirantis and S. Papageorgiou, "Cross-diffusion effects on chemical and biological pattern formation," Journal of Theoretical Biology, vol. 151, no. 3, pp. 289-311, 1991.
[27] N. Shigesada, K. Kawasaki, and E. Teramoto, "Spatial segregation of interacting species," Journal of Theoretical Biology, vol. 79, no. 1, pp. 83-99, 1979.

[28] G. Gambino, M. C. Lombardo, and M. Sammartino, "Pattern formation driven by cross-diffusion in a $2 \mathrm{~d}$ domain," Nonlinear Analysis-Real World Applications, vol. 14, pp. 1755-1779, 2013.

[29] L. A. Segel and J. L. Jackson, "Dissipative structure: an explanation and an ecological example," Journal of Theoretical Biology, vol. 37, no. 3, pp. 545-559, 1972.

[30] G.-Q. Sun, Z. Jin, L. Li, and B.-L. Li, "Self-organized wave pattern in a predator-prey model," Nonlinear Dynamics, vol. 60, no. 3, pp. 265-275, 2010.

[31] A. Sengupta, T. Kruppa, and H. Lowen, "Chemotactic predator-prey dynamics," Physical Review E, vol. 83, no. 3, Article ID 031914, 2011.

[32] G.-Q. Sun, Z. Jin, Q.-X. Liu, and L. Li, "Dynamical complexity of a spatial predator-prey model with migration," Ecological Modelling, vol. 219, no. 1-2, pp. 248-255, 2008.

[33] S. A. Levin and L. A. Segel, "Hypothesis for origin of planktonic patchiness," Nature, vol. 259, no. 5545, p. 659, 1976.

[34] W. Wang, X. Gao, Y. Cai, H. Shi, and S. Fu, "Turing patterns in a diffusive epidemic model with saturated infection force," Journal of the Franklin Institute, vol. 355, no. 15, pp. 7226-7245, 2018.

[35] R. Han and B. Dai, "Spatiotemporal pattern formation and selection induced by nonlinear cross-diffusion in a toxicphytoplankton-zooplankton model with Allee effect," Nonlinear Analysis: Real World Applications, vol. 45, pp. 822-853, 2019.

[36] S. Wu and Y. Song, "Spatiotemporal dynamics of a diffusive predator-prey model with nonlocal effect and delay," Communications in Nonlinear Science and Numerical Simulation, vol. 89, Article ID 105310, 2020.

[37] X. Cao and W. H. Jiang, "Double zero singularity and spatiotemporal patterns in a diffusive predator-prey model with nonlocal prey competition," Discrete and Continuous Dynamical Systems-Series B, vol. 25, pp. 3461-3489, 2017.

[38] M. C. Cross and P. C. Hohenberg, "Pattern formation outside of equilibrium," Reviews of Modern Physics, vol. 65, no. 3, pp. 851-1112, 1993.

[39] Q. Ouyang, Pattern Formation in the Reaction-Diffusion Systems, Shanghai Sci.-Tech. Education Publishing House, Shanghai, China, 2000.

[40] J. S. Wang, Y. P. Wu, L. Li et al., "Effect of mobility and predator switching on the dynamical behavior of a predator-prey model," Chaos Solitons And Fractals, vol. 132, Article ID 109584, 2020.

[41] C. Y. Wang, L. L. Chang, and H. F. Liu, "Spatial patterns of a predator-prey system of Leslie type with time delay," PLoS One, vol. 11, no. 3, p. e0150503, 2016.

[42] T. D. Frank, "Formal derivation of Lotka-Volterra-Haken amplitude equations of task-related brain activity in multiple, consecutively performed tasks," International Journal of Bifurcation and Chaos, vol. 26, no. 10, Article ID 1650164, 2016.

[43] Q. Ouyang, Introduction to Nonlinear Science and Pattern Dynamics, Peking University Press, Beijing, China, 2010.

[44] T. Reichenbach, M. Mobilia, and E. Frey, "Mobility promotes and jeopardizes biodiversity in rock-paper-scissors games," Nature, vol. 448, no. 7157, pp. 1046-1049, 2007.

[45] G. Q. Sun, Z. Y. Wu, Z. Wang et al., "Influence of isolation degree of spatial patterns on persistence of populations," Nonlinear Dynamics, vol. 83, no. 1-2, pp. 811-819, 2016. 
[46] G.-Q. Sun, C.-H. Wang, L.-L. Chang, Y.-P. Wu, L. Li, and Z. Jin, "Effects of feedback regulation on vegetation patterns in semi-arid environments," Applied Mathematical Modelling, vol. 61 , pp. 200-215, 2018.

[47] G. Q. Sun, M. Jusup, and Z. Jin, "Pattern transitions in spatial epidemics: mechanisms and emergent properties," Physics of Life Reviews, vol. 778, pp. 1-31, 2016.

[48] Z.-G. Guo, G.-Q. Sun, Z. Wang, Z. Jin, L. Li, and C. Li, "Spatial dynamics of an epidemic model with nonlocal infection," Applied Mathematics and Computation, vol. 377, Article ID 125158, 2020.

[49] G. Q. Sun, S. L. Wang, Q. Ren et al., "Effects of time delay and space on herbivore dynamics: linking inducible defenses of plants to herbivore outbreak," Scientific Reports, vol. 5, Article ID 11246, 2015. 